\author{
Hazem A. Attia \\ Ah1113@yahoo.com \\ Department of Mathematics \\ College of Science \\ Al-Qasseem University \\ P.O. Box 237 \\ Buraidah 81999, KSA
}

On leave from: Dept. of Eng. Math. and Physics Faculty of Engineering, El-Fayoum University, El-Fayoum, Egypt

\section{Dynamic Simulation of Constrained Mechanical Systems Using Recursive Projection Algorithm}

In this paper, the dynamic simulation of constrained mechanical systems that are interconnected of rigid bodies is studied using projection recursive algorithm. The method uses the concepts of linear and angular momentums to generate the rigid body equations of motion in terms of the Cartesian coordinates of a dynamically equivalent constrained system of particles, without introducing any rotational coordinates and the corresponding rotational transformation matrix. Closed-chain system is transformed to open-chain by cutting suitable kinematical joints and introducing cut-joint constraints. For the resulting open-chain system, the equations of motion are generated recursively along the serial chains. An example is chosen to demonstrate the generality and simplicity of the developed formulation.

Keywords: Dynamic simulation, equations of motion, recursive projection, mechanical systems, mechanisms

\section{Introduction}

There are different formulations for the dynamic analysis of spatial mechanisms which vary in the system of coordinates used and in the way they introduce kinematical constraint equations (Denavit and Hartenberg, 1955, Sheth and Uicker, 1972, Orlandea et al., 1977, Nikravesh, 1988, and De Jalon, 1994). Each formulation has its own advantages and disadvantages depending on the application and the needs. Some formulations are developed using a two-step transformation which leads to a simple and reduced system of equations. One method (Kim and Vanderploeg, 1986 and Nikravesh and Gim, 1989) and uses initially the absolute coordinate formulation where the location of each rigid body in the system is described in terms of a set of translational and rotational coordinates. Then, the equations of motion are transformed to a reduced set in terms of the relative joint variables. Another method uses initially the point coordinate formulation in which a dynamically equivalent constrained system of particles replaces the rigid bodies (De Jalon et al., 1986, Attia, 1993, Nikravesh and Attia, 1994, and Attia, 1998). The global motion of the constrained system of particles together with the constraints imposed upon them represent both the translational and rotational motions of the rigid body. The external forces and couples acting on the body are distributed over the system of particles. Then, the equations of motion which are expressed in terms of the Cartesian coordinates of the particles are re-derived in terms of the relative joint variables. The main disadvantage of this two-step transformation is the necessity to transform at every time step from the joint variables to the original system which is time consuming.

A recursive dynamical formulation for the dynamic analysis of planar mechanisms is presented by Attia (2004). The concepts of linear and angular momentum are used to write the rigid body dynamical equations without the need to distribute the external forces and couples over the particles. The method can be applied to recursively generate the equations of motion for open and/or closedchain systems.

In this paper, the dynamic simulation of constrained mechanical systems that are interconnected of rigid bodies is studied using projection recursive algorithm. The method is based upon the idea of replacing the rigid body by its dynamically equivalent

Paper accepted September, 2005. Technical Editor: Atila P. Silva Freire. constrained system of particles discussed in (De Jalon et al., 1986, Attia, 1993, Nikravesh and Attia, 1994, Attia, 1998 and Attia, 2004) with essential modifications and improvements. The concepts of the linear and angular momentums are used to formulate the rigid body dynamical equations. However, they are expressed in terms of the rectangular Cartesian coordinates of the equivalent constrained system of particles. This groups the advantages of the automatic elimination of the unknown internal forces as in Newton-Euler formulation and results in a reduced system of differential-algebraic equations. Some useful geometrical relationships are used to obtain a reduced dynamically equivalent constrained system of particles.

For the closed-chain system, the system is transformed to openchain system by cutting suitable kinematical joints and introducing the cut-joint kinematical constraints. For the resulting open-chain system, the equations of motion are generated recursively along the serial chains instead of the matrix formulation derived in (De Jalon et al., 1986, Attia, 1993, Nikravesh and Attia, 1994, and Attia, 1998). Most of the kinematical constraints due to the kinematical joints are automatically eliminated by properly locating the equivalent particles. Examples are chosen to demonstrate the generality and simplicity of the proposed method.

\section{The Dynamic Formulation}

\section{Construction of the Equivalent System of Particles}

A rigid body and its dynamically equivalent constrained system of particles should have the same mass, the position of the centre of mass and the inertia tensor with respect to a body attached coordinate frame which results in ten conditions in the spatial case. The choice of four particles (not all are laying in the same plane) results in 16 unknowns (4 masses +12 coordinates) that should satisfy the ten conditions. However, this choice will lead to the solution of nonlinear algebraic equations due to the quadratic form of the second moments and also it does not give the freedom to choose all the particles in important places in the mechanisms. A system of ten particles will lead to the solution of ten linear algebraic equations in ten unknown masses. Also it gives the freedom of positioning the particles on the bodies in accordance with the joints that connect the bodies in order to reduce the number of particles and consequently eliminates some geometric and kinematical constraints. Therefore, a system of ten particles is chosen to replace the rigid body as shown in Fig. 1. It constitutes 
four particles $1, \ldots, 4$, which are denoted as primary particles and additional six particles $5, . ., 10$, which are denoted as secondary particles. Each secondary particle is located at the middle point between a pair of primary particles in order to facilitate the elimination of its acceleration components and, in turn, reduce the unknown motion variables. The mass distributions to points must satisfy the following conditions

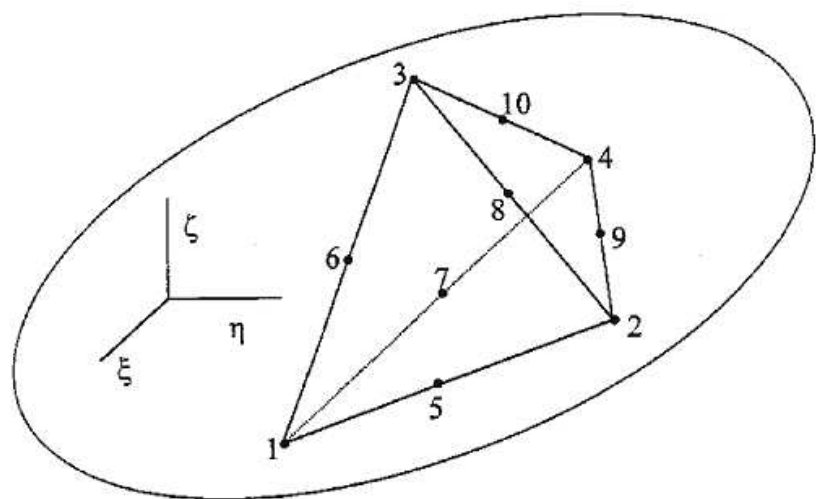

Figure 1. The rigid body system with the equivalent system of ten particles.

$$
\begin{gathered}
m=\sum_{i=1}^{10} m_{i} \\
m_{G}=\sum_{i=1}^{10} m_{i} \bar{r}_{i} \\
I_{\xi \xi}=\sum_{i=1}^{10} m_{i}\left(\zeta_{i}^{2}+\eta_{i}^{2}\right) \\
I_{\eta \eta}=\sum_{i=1}^{10} m_{i}\left(\zeta_{i}^{2}+\xi_{i}^{2}\right) \\
I_{\zeta \zeta}=\sum_{i=1}^{10} m_{i}\left(\xi_{i}^{2}+\eta_{i}^{2}\right) \\
I_{\xi \eta}=\sum_{i=1}^{10} m_{i} \xi_{i} \eta_{i} \\
I_{\xi \zeta}=\sum_{i=1}^{10} m_{i} \xi_{i} \zeta_{i} \\
I_{\zeta \eta}=\sum_{i=1}^{10} m_{i} \zeta_{i} \eta_{i}
\end{gathered}
$$

where $m$ is the mass of the body, $\bar{r}_{G}$ is the position vector of the centre of mass of the body with respect to the body attached coordinate frame, $I_{\xi \xi}, I_{\eta \eta}, I_{\zeta \zeta}$ are the moments of inertia of the body with respect to the body attached coordinate frame, $I_{\xi \eta}, I_{\xi \zeta}$, $I_{\zeta \eta}$ are the products of inertia of the body with respect to the body attached coordinate frame, $m_{i}$ is the mass of particle $\mathrm{i}$, and $\bar{r}_{i}$ is the position vector of particle $i$ with respect to the attached coordinate frame. Equation (1) represents a 10x10 linear system of algebraic equations in 10 unknown masses of the primary and secondary particles. At the same time, the coordinates of the particles can be chosen arbitrarily, which gives the advantage of the automatic elimination of the kinematical constraints due to some mechanical joints. Also it allows for two adjacent rigid bodies to contribute to the mass concentrated at the joint connecting them which reduces the total number of particles replacing the whole system.

Equations of Motion of a Single Rigid Body in Spatial Motion

Consider a rigid body which is acted upon by external forces and force couples. The rigid body is replaced by an equivalent system of ten particles. The distances between the ten particles are invariants as a result of the internal forces existing between them. The vector sum of these unknown internal forces or also the vector sum of their moments about any point equals zero by the law of action and reaction (Goldstein, 1950). Then, the linear momentum equation for the whole system of particles yields,

$$
\boldsymbol{R}=\sum_{i=1}^{10} m_{i} \ddot{r}_{i}
$$

where $\boldsymbol{R}$ is the vector sum of the external forces acting on the rigid body and $\ddot{r}_{i}$ is the acceleration vector of particle $i$. Also, the angular momentum equation for the whole system of particles with respect to particle 1 results in (Goldstein, 1950)

$$
\boldsymbol{G}_{1}=\sum_{i=2}^{10} m_{i} \boldsymbol{r}_{i, 1} x \ddot{\boldsymbol{r}}_{i}=\sum_{i=2}^{10} m_{i} \tilde{\boldsymbol{r}}_{i, 1} \ddot{\boldsymbol{r}}_{i}
$$

where $G_{1}$ is the vector sum of the moments of the external forces and force couples acting on the body with respect to particle $1, \boldsymbol{r}_{i, 1}$ is the relative position vector between particles $i$ and 1 , and $\tilde{r}_{i, 1}$ is the $3 \times 3$ skew-symmetric tensor providing the vector product. The distance constraints between the ten particles are given as (see Fig. 1)

$$
\begin{gathered}
\boldsymbol{r}_{2,1}^{T} \boldsymbol{r}_{2,1}-d_{2,1}^{2}=0 \\
\boldsymbol{r}_{4,1}^{T} \boldsymbol{r}_{4,1}-d_{4,1}^{2}=0 \\
\boldsymbol{r}_{4,2}^{T} \boldsymbol{r}_{4,2}-d_{4,2}^{2}=0 \\
\boldsymbol{r}_{3,1}^{T} \boldsymbol{r}_{3,1}-d_{3,1}^{2}=0 \\
\boldsymbol{r}_{3,2}^{T} \boldsymbol{r}_{3,2}-d_{3,2}^{2}=0 \\
\boldsymbol{r}_{3,4}^{T} \boldsymbol{r}_{3,4}-d_{3,4}^{2}=0 \\
\boldsymbol{r}_{5}-\left(\boldsymbol{r}_{1}+\boldsymbol{r}_{2}\right) / 2=0 \\
\boldsymbol{r}_{6}-\left(\boldsymbol{r}_{1}+\boldsymbol{r}_{3}\right) / 2=0 \\
\boldsymbol{r}_{7}-\left(\boldsymbol{r}_{1}+\boldsymbol{r}_{4}\right) / 2=0 \\
\boldsymbol{r}_{8}-\left(\boldsymbol{r}_{2}+\boldsymbol{r}_{3}\right) / 2=0
\end{gathered}
$$




$$
\begin{aligned}
& \boldsymbol{r}_{9}-\left(\boldsymbol{r}_{2}+\boldsymbol{r}_{4}\right) / 2=0 \\
& \boldsymbol{r}_{10}-\left(\boldsymbol{r}_{3}+\boldsymbol{r}_{4}\right) / 2=0
\end{aligned}
$$

where the masses $m_{5}, m_{6}, m_{7}, m_{8}, m_{9}, m_{10}$ are located, respectively, at the middle point of masses $m_{1}$ and $m_{2}, m_{1}$ and $m_{3}, m_{1}$ and $m_{4}, m_{2}$ and $m_{3}, m_{2}$ and $m_{4}$, and $m_{3}$ and $m_{4}$. The equations of motion (2), (3) and (4) represent a system of differential-algebraic equations that can be solved to determine the unknown acceleration vectors $\ddot{r}_{i}$ of the particles at any instant of time. However, due to the large number of the geometric constraints the integration of these equations is inefficient. In the following section, some useful geometrical relationships are used to eliminate the majority of these constraints.

\section{The Reduced Form of the Equations of Motion of a Single} Rigid Body

The reduced form of the equations of motion can be achieved in two steps. First, the accelerations of the secondary particles and their unknown accelerations can be easily eliminated by substituting the constraint Eqs. (4.7) to (4.12) into Eqs. (2) and (3) to obtain

$$
\begin{gathered}
\boldsymbol{R}=\sum_{i=1}^{4} \bar{m}_{i} \ddot{\boldsymbol{r}}_{i} \\
\boldsymbol{G}_{1}=\sum_{i=1}^{4} \boldsymbol{A}_{i} \ddot{\boldsymbol{r}}_{i}
\end{gathered}
$$

where

$$
\begin{gathered}
\bar{m}_{i}=m_{i}+\sum_{j=1, j \neq i}^{4} \frac{1}{2} m_{i, j}, \\
\boldsymbol{A}_{i}=\overline{\bar{m}}_{i} \tilde{\boldsymbol{r}}_{i, 1}+\sum_{j=2, j \neq i}^{4} \frac{1}{4} m_{i, j} \tilde{\boldsymbol{r}}_{j, 1}, \\
\overline{\bar{m}}_{i}=m_{i}+\sum_{j=1, j \neq i}^{4} \frac{1}{4} m_{i, j},
\end{gathered}
$$

and where $m_{i, j}$ denotes the mass of the secondary particle that is located between the primary particles $\mathrm{i}$ and $\mathrm{j}\left(m_{1,2}=m_{5}, \ldots\right.$ etc.). Then, Eqs. (5) and (6) in addition to the remaining constraints Eqs. (4.1) to (4.6) represent the equations of motion for a single rigid body where only the accelerations of the primary particles stay.

A more reduced set of equations of motion can be derived by expressing the position vector of one of the primary particles in terms of the position vectors of the other three primary particles. We choose to express the coordinates of particle 3 in terms of the coordinates of particles 1,2, and 4 . As shown in Fig. 2, three invariant quantities $\bar{\lambda}, \bar{\mu}$, and $\bar{\tau}$ can be estimated with the aid of the constraint Eqs. (4.4) to (4.6) that fix the distances between particle 3 and particles 1,2 , and 4 , respectively. The invariant quantities take the form,

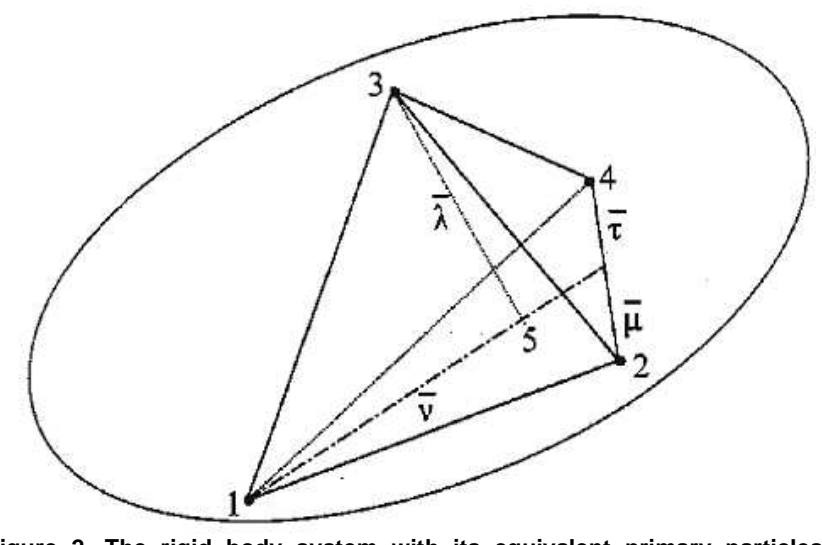

Figure 2. The rigid body system with its equivalent primary particles indicating the invariant quantities.

$$
\begin{gathered}
\bar{\lambda}=\mid \frac{\boldsymbol{r}_{3,1}^{T} \tilde{\boldsymbol{r}}_{2,1} \boldsymbol{r}_{4,1} \mid}{\left|\tilde{\boldsymbol{r}}_{2,1} \boldsymbol{r}_{4,1}\right|}, \\
\bar{v}=\sqrt{\left|\boldsymbol{r}_{3,1}\right|^{2}-\bar{\lambda}^{2}}, \\
\bar{\mu}=\frac{\left|\boldsymbol{r}_{4,2}\right|\left|\tilde{r}_{2,1} \boldsymbol{r}_{5,1}\right|}{\left|\tilde{r}_{5,1} \boldsymbol{r}_{4,2}\right|}, \\
\bar{\tau}=\left|\boldsymbol{r}_{4,2}\right|-\bar{\mu},
\end{gathered}
$$

where

$$
\boldsymbol{r}_{5,1}=\boldsymbol{r}_{3,1}-\bar{\lambda} \frac{\tilde{\boldsymbol{r}}_{2,1} \boldsymbol{r}_{4,1}}{\left|\tilde{\boldsymbol{r}}_{2,1} \boldsymbol{r}_{4,1}\right|}
$$

Knowing the initial Cartesian coordinates of the primary particles, the invariant quantities are determined using Eqs. (10). In terms of these invariant quantities, the position vector of particle 3 is expressed as

$$
\boldsymbol{r}_{3}=\boldsymbol{r}_{1}+\bar{\lambda} \frac{\tilde{\boldsymbol{r}}_{2,1} \boldsymbol{r}_{4,1}}{\left|\tilde{\boldsymbol{r}}_{2,1} \boldsymbol{r}_{4,1}\right|}+\bar{v} \frac{\bar{\mu} \boldsymbol{r}_{4,1}+\bar{\tau} \boldsymbol{r}_{2,1}}{\left|\bar{\mu} \boldsymbol{r}_{4,1}+\bar{\tau} \boldsymbol{r}_{2,1}\right|},
$$

Since the quantities in the denominators in the right hand side of Eq. (11) are invariants we can rearrange the terms and obtain the simpler form

$$
r_{3}=r_{1}+\lambda \tilde{r}_{2,1} r_{4,1}+\mu r_{4,1}+\tau r_{2,1}
$$

where

$$
\lambda=\frac{\bar{\lambda}}{\left|\tilde{r}_{2,1} \boldsymbol{r}_{4,1}\right|}, \mu=\frac{\bar{v} \bar{\mu}}{\left|\bar{\mu} r_{4,1}+\bar{\tau} r_{2,1}\right|}, \tau=\frac{\bar{v} \tau}{\left|\bar{\mu} r_{4,1}+\bar{\tau} r_{2,1}\right|},
$$

The corresponding velocity and acceleration vectors of particle 3 are estimated using the first and second time differentiations of Eq. (12) respectively which result in the following forms, 


$$
\begin{gathered}
\dot{\boldsymbol{r}}_{3}=\dot{\boldsymbol{r}}_{1}+\lambda\left(\tilde{\boldsymbol{r}}_{2,1} \dot{\boldsymbol{r}}_{4,1}+\dot{\tilde{r}}_{2,1} \boldsymbol{r}_{4,1}\right)+\mu \dot{\boldsymbol{r}}_{4,1}+\tau \dot{\boldsymbol{r}}_{2,1}, \\
\ddot{\boldsymbol{r}}_{3}=\ddot{\boldsymbol{r}}_{1}+\lambda\left(\tilde{\boldsymbol{r}}_{2,1} \ddot{\boldsymbol{r}}_{4,1}+\ddot{\widetilde{\boldsymbol{r}}}_{2,1} \boldsymbol{r}_{4,1}+2 \dot{\widetilde{r}}_{2,1} \dot{\boldsymbol{r}}_{4,1}\right)+\mu \ddot{\boldsymbol{r}}_{4,1}+\tau \ddot{\boldsymbol{r}}_{2,1},
\end{gathered}
$$

Equation (14) expresses the unknown acceleration vector of particle 3 in terms of the acceleration vectors of the other primary particles which eliminates the constraint Eqs. (4.4) to (4.6). Equation (14) can be put in the more convenient form,

$$
\ddot{r}_{3}=\left(1-\mu-\tau+\lambda \tilde{r}_{4,2}\right) \ddot{r}_{1}+\left(\tau-\lambda \tilde{r}_{4,1}\right) \ddot{r}_{2}+\left(\mu+\lambda \tilde{r}_{2,1}\right) \ddot{r}_{4},
$$

Substituting the derived acceleration vector of particle 3 from Eq. (15) into Eqs. (5) and (6), then the differential equations of motion take the modified form

$$
\begin{aligned}
\boldsymbol{R}=\left\{\bar{m}_{1}\right. & \left.+\bar{m}_{3}\left(1-\mu-\tau+\lambda \tilde{\boldsymbol{r}}_{4,2}\right)\right\} \ddot{\boldsymbol{r}}_{1}+\left\{\bar{m}_{2}+\bar{m}_{3}\left(\tau-\lambda \tilde{\boldsymbol{r}}_{4,1}\right)\right\} \ddot{\boldsymbol{r}}_{2} \\
& +\left\{\bar{m}_{4}+\bar{m}_{3}\left(\mu+\lambda \tilde{\boldsymbol{r}}_{2,1}\right)\right\} \ddot{r}_{4}+2 \lambda \bar{m}_{3} \dot{\tilde{r}}_{2,1} \dot{r}_{4,1} \\
\boldsymbol{G}_{1}=\left\{\boldsymbol{A}_{1}\right. & \left.+\boldsymbol{A}_{3}\left(1-\mu-\tau+\lambda \tilde{\boldsymbol{r}}_{4,2}\right)\right\} \ddot{\boldsymbol{r}}_{1}+\left\{\boldsymbol{A}_{2}+\boldsymbol{A}_{3}\left(\tau-\lambda \tilde{\boldsymbol{r}}_{4,1}\right)\right\} \ddot{\boldsymbol{r}}_{2} \\
& +\left\{\boldsymbol{A}_{4}+\boldsymbol{A}_{3}\left(\mu+\lambda \tilde{\boldsymbol{r}}_{2,1}\right)\right\} \ddot{r}_{4}+2 \lambda \boldsymbol{A}_{3} \dot{\tilde{r}}_{2,1} \dot{\boldsymbol{r}}_{4,1}
\end{aligned}
$$

Equations (16) and (17) in addition to the constraint Eqs. (4.1) to (4.3) represent the equations of motion of a single floating rigid body in spatial motion. It can be solved at every time step to determine the unknown acceleration components of particles 1, 2, and 4. Consequently, Eq. (15) can be used to determine the acceleration components of particle 3 . The acceleration components of the particles are integrated numerically knowing their Cartesian coordinates and velocities at a certain time to determine the positions and velocities for the next time step. Gear's method (Gear, 1988 ) for the numerical integration of differential-algebraic equations is used to overcome the instability problem resulting during the modelling process of constraint mechanical systems. The motion of the particles determines completely the translational and rotational motion of the rigid body. If the rigid body is rotating about a fixed point, then particle 1 may be located at the centre of this joint. In this case, Eq. (17) and Eqs. (4.1) to (4.3) are used to solve for the unknown Cartesian accelerations of particles 2 and 4. Equation (16) can be solved to determine the unknown reaction forces at the joint $N_{l}$ as,

$$
\begin{gathered}
\boldsymbol{N}_{1}=\left\{\bar{m}_{1}+\bar{m}_{3}\left(1-\mu-\tau+\lambda \tilde{\boldsymbol{r}}_{4,2}\right)\right\} \ddot{r}_{1}+\left\{\bar{m}_{2}+\bar{m}_{3}\left(\tau-\lambda \tilde{\boldsymbol{r}}_{4,1}\right)\right\} \ddot{r}_{2} \\
+\left\{\bar{m}_{4}+\bar{m}_{3}\left(\mu+\lambda \tilde{\boldsymbol{r}}_{2,1}\right)\right\} \ddot{\boldsymbol{r}}_{4}+2 \lambda \bar{m}_{3} \dot{\tilde{r}}_{2,1} \dot{\boldsymbol{r}}_{4,1}-\boldsymbol{R}
\end{gathered}
$$

If the rigid body is rotating about a fixed axis, then particles 1 and 2 can be located along the axis of the joint to define its direction. Then, to solve for the unknown acceleration vector of particle 4, the constraint equations (4.2) and (4.3) can be used in addition to one scalar moment equation that is generated by taking the projection of the vectors in Eq. (17) along the direction of the fixed axis. Then, Eq. (16) may be used to get the reactions at the axis of the revolute joint.

\section{Equations of Motion of a Serial Chain of Rigid Bodies}

In the matrix formulations of multibody systems, the system is divided into individual bodies and the equations of motion for each body are written in matrix form. Therefore, the equations of motion for the whole system are derived by grouping and overlapping the mass matrices of all rigid bodies resulting in the overall mass matrix which characterizes the matrix formulation. On the other hand, in the recursive formulations, no separation between bodies is done, however, different serial chains are constructed. The force and moment equations are written for each serial chain progressing from one body to the adjacent body in the chain in a recursive manner.

\section{Case of All are Spherical Joints:}

Figure 3 shows a serial chain of $N$ rigid bodies connected by spherical joints with the equivalent system of $(3 \mathrm{~N}+1)$ particles where connected particles are unified from both bodies.

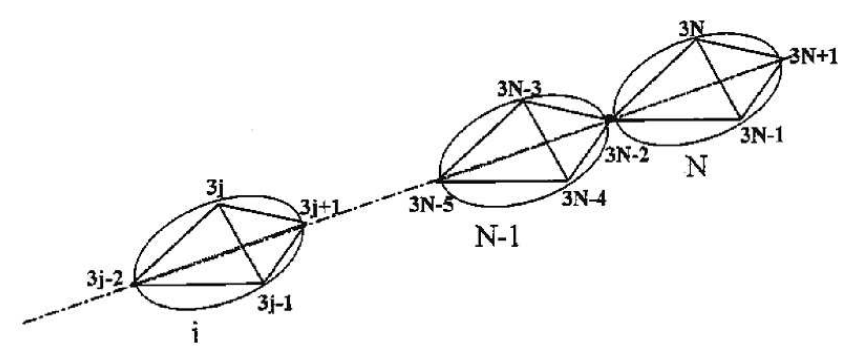

Figure 3. Serial chain of $\mathbf{N}$ rigid bodies with the equivalent system of primary particles.

Step 1: For the last body $N$ in the chain, the equations of motion are derived in a similar way as Eq. (17) and Eqs. (4.1) to (4.3) of a single rigid body. The angular momentum equation takes the form

$$
\begin{aligned}
\boldsymbol{G}_{N, 3 N-2}= & \left\{\boldsymbol{A}_{3 N-2}+\boldsymbol{A}_{3 N}\left(1-\mu_{N}-\tau_{N}+\lambda_{N} \tilde{\boldsymbol{r}}_{3 N+1,3 N-1}\right)\right\} \ddot{\boldsymbol{r}}_{3 N-2} \\
+ & \left\{\boldsymbol{A}_{3 N-1}+\boldsymbol{A}_{3 N}\left(\tau_{N}-\lambda_{N} \tilde{\boldsymbol{r}}_{3 N+1,3 N-2}\right)\right\} \ddot{r}_{3 N-1} \\
+ & \left\{\boldsymbol{A}_{3 N+1}+\boldsymbol{A}_{3 N}\left(\mu_{N}+\lambda_{N} \tilde{\boldsymbol{r}}_{3 N-1,3 N-2}\right)\right\} \ddot{\boldsymbol{r}}_{3 N+1} \\
& \quad+2 \lambda_{N} \boldsymbol{A}_{3 N} \dot{\tilde{\boldsymbol{r}}}_{3 N-1,3 N-2} \dot{\boldsymbol{r}}_{3 N+!, 3 N-2}
\end{aligned}
$$

where

$$
\begin{gathered}
\boldsymbol{A}_{3 N}=\overline{\bar{m}}_{3 N} \tilde{\boldsymbol{r}}_{3 N, 3 N-2}+\sum_{i=3 N-1, i \neq 3 N}^{3 N+1} \frac{1}{4} m_{3 N, i} \tilde{\boldsymbol{r}}_{i, 3 N-2}, \\
\overline{\bar{m}}_{3 N}=m_{3 N}+\sum_{i=3 N-2, i \neq 3 N}^{3 N+1} \frac{1}{4} m_{3 N, i},
\end{gathered}
$$

where $\boldsymbol{G}_{N, 3 N-2}$ is the sum of the moments of the external forces and force couples acting on body $N$ with respect to the location of particle $3 N-2$. The acceleration equations of the distance constraint between primary particles belonging to body $N$ are given as

$$
\begin{aligned}
& \boldsymbol{r}_{3 N-2,3 N-1}^{T} \ddot{r}_{3 N-2}+\boldsymbol{r}_{3 N-1,3 N-2}^{T} \ddot{r}_{3 N-1}=-\dot{\boldsymbol{r}}_{3 N-1,3 N-2}^{T} \dot{\boldsymbol{r}}_{3 N-1,3 N-2} \\
& \boldsymbol{r}_{3 N-2,3 N+1}^{T} \ddot{r}_{3 N-2}+\boldsymbol{r}_{3 N+1,3 N-2}^{T} \ddot{r}_{3 N+1}=-\dot{\boldsymbol{r}}_{3 N+1,3 N-2}^{T} \dot{r}_{3 N+1,3 N-2} \\
& \boldsymbol{r}_{3 N-1,3 N+1}^{T} \ddot{\boldsymbol{r}}_{3 N-1}+\boldsymbol{r}_{3 N+1,3 N-1}^{T} \ddot{\boldsymbol{r}}_{3 N+1}=-\dot{\boldsymbol{r}}_{3 N+1,3 N-1}^{T} \dot{\boldsymbol{r}}_{3 N+1,3 N-1}
\end{aligned}
$$

Step 2: Addition of one more body in the chain leads to the inclusion of an angular momentum vector equation that takes into consideration the contributions of all the ascending bodies in the chain together with three distance constraint equations between the 
particles belonging to this body. These six scalar equations are appended to the equations of motion derived for the leading bodies in the chain. For body $j$, the appended equations of motion take the form

$$
\begin{aligned}
\sum_{k=j}^{N} \boldsymbol{G}_{k, 3 j-2}= & \sum_{k=j}^{N}\left\{\boldsymbol{A}_{3 k-2}+\boldsymbol{A}_{3 k}\left(1-\mu_{k}-\tau_{k}+\lambda_{k} \tilde{\boldsymbol{r}}_{3 k+1,3 k-1}\right)\right\} \ddot{\boldsymbol{r}}_{3 k-2} \\
& +\left\{\boldsymbol{A}_{3 k-1}+\boldsymbol{A}_{3 k}\left(\tau_{k}-\lambda_{k} \tilde{\boldsymbol{r}}_{3 k+1,3 k-2}\right)\right\} \ddot{\boldsymbol{r}}_{3 k-1} \\
& +\left\{\boldsymbol{A}_{3 k+1}+\boldsymbol{A}_{3 k}\left(\mu_{k}+\lambda_{k} \tilde{\boldsymbol{r}}_{3 k-1,3 k-2}\right)\right\} \ddot{\boldsymbol{r}}_{3 k+1} \\
& +2 \lambda_{k} \boldsymbol{A}_{3 k} \dot{\tilde{\boldsymbol{r}}}_{3 k-1,3 k-2} \dot{\boldsymbol{r}}_{3 k+!, 3 k-2}
\end{aligned}
$$

where

$$
\begin{gathered}
A_{3 k}=\overline{\bar{m}}_{3 k} \tilde{\boldsymbol{r}}_{3 k, 3 j-2}+\sum_{i=3 k-1, i \neq 3 k}^{3 k+1} \frac{1}{4} m_{3 k, i} \tilde{\boldsymbol{r}}_{i, 3 k-2}, \\
\overline{\bar{m}}_{3 k}=m_{3 k}+\sum_{i=3 k-2, i \neq 3 k}^{3 k+1} \frac{1}{4} m_{3 k, i}, \\
\boldsymbol{r}_{3 j-2,3 j-1}^{T} \ddot{\boldsymbol{r}}_{3 j-2}+\boldsymbol{r}_{3 j-1,3 j-2}^{T} \ddot{\boldsymbol{r}}_{3 j-1}=-\dot{\boldsymbol{r}}_{3 j-1,3 j-2}^{T} \dot{\boldsymbol{r}}_{3 j-1,3 j-2} \\
\boldsymbol{r}_{3 j-2,3 j+1}^{T} \ddot{\boldsymbol{r}}_{3 j-2}+\boldsymbol{r}_{3 j+1,3 j-2}^{T} \ddot{\boldsymbol{r}}_{3 j+1}=-\dot{\boldsymbol{r}}_{3 j+1,3 j-2}^{T} \dot{\boldsymbol{r}}_{3 j+1,3 j-2} \\
\boldsymbol{r}_{3 j-1,3 j+1}^{T} \ddot{\boldsymbol{r}}_{3 j-1}+\boldsymbol{r}_{3 j+1,3 j-1}^{T} \ddot{\boldsymbol{r}}_{3 j+1}=-\dot{\boldsymbol{r}}_{3 j+1,3 j-1}^{T} \dot{\boldsymbol{r}}_{3 j+1,3 j-1}
\end{gathered}
$$

where according to Eq. (15),

$$
\begin{aligned}
\ddot{\boldsymbol{r}}_{3 k}=\left(1-\mu_{k}-\tau_{k}\right. & \left.+\lambda_{k} \tilde{\boldsymbol{r}}_{3 k+1,3 k-1}\right) \ddot{r}_{3 k-2}+\left(\tau_{k}-\lambda_{k} \tilde{\boldsymbol{r}}_{3 k+1,3 k-2}\right) \ddot{r}_{3 k-1} \\
& +\left(\mu_{k}+\lambda_{k} \tilde{\boldsymbol{r}}_{3 k-1,3 k-2}\right) \ddot{\boldsymbol{r}}_{3 k+1},
\end{aligned}
$$

If body $j$ is the floating base body in the chain then, three linear momentum equations, similar to Eq. (16), are required to solve for the unknown acceleration components of particle 1 . These linear momentum equations equate the sum of the external forces acting on all the bodies in the chain to the time rate of change of the vectors of linear momentum of all the equivalent particles that replace the chain which take the form

$$
\begin{aligned}
\sum_{k=j}^{N} \boldsymbol{R}= & \sum_{k=j}^{N}\left\{\bar{m}_{3 k-2}+\bar{m}_{3 k}\left(1-\mu_{k}-\tau_{k}+\lambda_{k} \tilde{\boldsymbol{r}}_{3 k+1,3 k-1}\right)\right\} \ddot{\boldsymbol{r}}_{3 k-2} \\
& +\left\{\bar{m}_{3 k-1}+\bar{m}_{3 k}\left(\tau_{k}-\lambda_{k} \tilde{\boldsymbol{r}}_{3 k+1,3 k-2}\right)\right\} \ddot{r}_{3 k-1} \\
& +\left\{\bar{m}_{3 k+1}+\bar{m}_{3 k}\left(\mu_{k}+\lambda_{k} \tilde{\boldsymbol{r}}_{3 k-1,3 k-2}\right)\right\} \ddot{r}_{3 k+1} \\
& +2 \lambda_{k} \bar{m}_{3 k} \dot{\tilde{r}}_{3 k-1,3 k-2} \dot{r}_{3 k+1,3 k-2}
\end{aligned}
$$

where

$$
\bar{m}_{3 k}=m_{3 k}+\sum_{i=3 k-2, i \neq 3 k}^{3 k+1} \frac{1}{2} m_{3 k, i} .
$$

In general, for a serial chain of $N$ bodies, an equivalent system of $(3 N+1)$ primary particles and $6 N$ secondary particles is first constructed. Then, by eliminating all the accelerations of the secondary particles and $N$ primary particles, we are left with $2 N+1$ accelerations of the particles and consequently, $6 N+3$ unknown acceleration components. To solve for these unknowns, $3 N$ angular momentum equations can be generated recursively along the chain together with $3 N$ distance constraints between the particles located on each body. Finally, three linear momentum equations can be used to solve for the unknown acceleration components of particle 1 if body 1 is floating or for the unknown reaction forces if there is a fixation at point 1 .

Step 3: If body $j$ is connected to body $j-1$ by a revolute joint, then we take the projection of all the moment vectors in Eq. (20) along the axis of the joint which is defined by two particles from both bodies that are commonly located on it. Two additional distance constraints, that fix the distances between the remaining fourth particle and the other two particles along the axis of the joint, together with the angular momentum equation can be used to solve for the acceleration vector of the fourth particle on body $j$.

Step 4: If bodies $j$ and $j-1$ in a serial chain are connected by a prismatic joint, then particles $3 j-5,3 j-4,3 j-3$, and $3 j-2$ are located on body $j-1$ while particles $3 j-1,3 j, 3 j+1$, and $3 j+2$ are assigned to body $j$. Particles $3 j-5$ and $3 j-2$ on body $j-1$ and particles $3 j-1$ and $3 j+2$ on body $j$ are arbitrarily located along the axis of the prismatic joint. To obtain the equations of motion for body $j$, one force equation can be written by taking the projection of all the vectors in Eq. (20) along the axis of the prismatic joint together with the distance constraint Eqs. (21.2)-(21.3). Moreover, five independent kinematical constraint equations associated with the prismatic joint are included and take the form,

$$
\begin{aligned}
& \left(\boldsymbol{r}_{3 j-5}-\boldsymbol{r}_{3 j-2}\right) x\left(\boldsymbol{r}_{3 j-1}-\boldsymbol{r}_{3 j+2}\right)=0, \\
& \left(\boldsymbol{r}_{3 j-5}-\boldsymbol{r}_{3 j-2}\right) x\left(\boldsymbol{r}_{3 j-1}-\boldsymbol{r}_{3 j-2}\right)=0,
\end{aligned}
$$

$\left(\boldsymbol{r}_{3 j-4,3 j-5}-\frac{\boldsymbol{r}_{3 j-4,3 j-5}^{T} \boldsymbol{r}_{3 j-2,3 j-5}}{\left|\boldsymbol{r}_{3 j-2,3 j-5}\right|}\right)^{T}\left(\boldsymbol{r}_{3 j, 3 j-1}-\frac{\boldsymbol{r}_{3 j, 3 j-1}^{T} \boldsymbol{r}_{3 j+2,3 j-1}}{\left|\boldsymbol{r}_{3 j+2,3 j-1}\right|}\right)=0$

Therefore, for a preceding body $h$ in the chain the moment equation is generated recursively along the serial chain as addressed above which take the form,

$$
\begin{aligned}
& \sum_{k=h}^{N} \boldsymbol{G}_{k, 3 h-2}= \sum_{k=h}^{j-1}\left\{\boldsymbol{A}_{3 k-2}+\boldsymbol{A}_{3 k}\left(1-\mu_{k}-\tau_{k}+\lambda_{k} \tilde{\boldsymbol{r}}_{3 k+1,3 k-1}\right)\right\} \ddot{\boldsymbol{r}}_{3 k-2} \\
&++\left\{\boldsymbol{A}_{3 k-1}+\boldsymbol{A}_{3 k}\left(\tau_{k}-\lambda_{k} \tilde{\boldsymbol{r}}_{3 k+1,3 k-2}\right)\right\} \ddot{r}_{3 k-1} \\
&+\left.+\boldsymbol{A}_{3 k+1}+\boldsymbol{A}_{3 k}\left(\mu_{k}+\lambda_{k} \tilde{\boldsymbol{r}}_{3 k-1,3 k-2}\right)\right\} \ddot{\boldsymbol{r}}_{3 k+1} \\
&+2 \lambda_{k} \boldsymbol{A}_{3 k} \dot{\tilde{\boldsymbol{r}}}_{3 k-1,3 k-2} \dot{\boldsymbol{r}}_{3 k+!, 3 k-2} \\
&+\sum_{k=j}^{N}\left\{\boldsymbol{A}_{3 k-1}+\boldsymbol{A}_{3 k+1}\left(1-\mu_{k}-\tau_{k}+\lambda_{k} \tilde{\boldsymbol{r}}_{3 k+2,3 k}\right)\right\} \ddot{r}_{3 k-1} \\
&+ \\
&+\left\{\boldsymbol{A}_{3 k}+\boldsymbol{A}_{3 k+1}\left(\tau_{k}-\lambda_{k} \tilde{\boldsymbol{r}}_{3 k+2,3 k-1}\right)\right\} \ddot{\boldsymbol{r}}_{3 k} \\
&+\left\{\boldsymbol{A}_{3 k+2}+\boldsymbol{A}_{3 k+1}\left(\mu_{k}+\lambda_{k} \tilde{\boldsymbol{r}}_{3 k, 3 k-1}\right)\right\} \ddot{\ddot{r}}_{3 k+2} \\
& \quad+2 \lambda_{k} \boldsymbol{A}_{3 k+1} \dot{\tilde{r}}_{3 k, 3 k-1} \dot{\boldsymbol{r}}_{3 k+2,3 k-1}
\end{aligned}
$$

If body $h$ is the floating base body in the chain, then a force equation, similar to Eq. (22), is written to solve for the unknown acceleration of particle 1 in the form, 


$$
\begin{aligned}
& \sum_{k=h}^{N} \boldsymbol{R}_{k}= \sum_{k=h}^{j-1}\left\{\bar{m}_{3 k-2}+\bar{m}_{3 k}\left(1-\mu_{k}-\tau_{k}+\lambda_{k} \tilde{\boldsymbol{r}}_{3 k+1,3 k-1}\right)\right\} \ddot{r}_{3 k-2} \\
&+\left\{\bar{m}_{3 k-1}+\bar{m}_{3 k}\left(\tau_{k}-\lambda_{k} \tilde{\boldsymbol{r}}_{3 k+1,3 k-2}\right)\right\} \ddot{\boldsymbol{r}}_{3 k-1} \\
&+\left\{\bar{m}_{3 K-1}+\bar{m}_{3 k}\left(\mu_{k}+\lambda_{k} \tilde{\boldsymbol{r}}_{3 k-1,3 k-2}\right)\right\} \ddot{\boldsymbol{r}}_{3 k+1} \\
&+2 \lambda_{k} \bar{m}_{3 k} \dot{\tilde{\boldsymbol{r}}}_{3 k-1,3 k-2} \dot{\boldsymbol{r}}_{3 k+1,3 k-2} \\
&+\sum_{k=j}^{N}\left\{\bar{m}_{3 k-1}+\bar{m}_{3 k+1}\left(1-\mu_{k}-\tau_{k}+\lambda_{k} \tilde{\boldsymbol{r}}_{3 k+2,3 k}\right)\right\} \ddot{\boldsymbol{r}}_{3 k-1} \\
&+\left\{\bar{m}_{3 k}+\bar{m}_{3 k+1}\left(\tau_{k}-\lambda_{k} \tilde{\boldsymbol{r}}_{3 k+2,3 k-1}\right)\right\} \ddot{\boldsymbol{r}}_{3 k} \\
&+\left\{\bar{m}_{3 k+2}+\bar{m}_{3 k+1}\left(\mu_{k}+\lambda_{k} \tilde{\boldsymbol{r}}_{3 k, 3 k-1}\right)\right\} \ddot{r}_{3 k+2} \\
& \quad+2 \lambda_{k} \bar{m}_{3 k+1} \dot{\tilde{r}}_{3 k, 3 k-1} \dot{\boldsymbol{r}}_{3 k+2,3 k-1}
\end{aligned}
$$

Similar treatment can be used in dealing with all other kinds of lower or higher-pair kinematical joints.

\section{Case of an Open-Chain or Closed-Chain}

In the case of an open-chain system or closed-chain system, it can be transformed to a system of serial chains by cutting suitable joints and consequently cut-joint constraints are introduced. In the case of a closed-chain system, the cut-joints avoids the need to introduce loop closure equations and the corresponding loop closure constraint forces and then allows the use of the laws of momentum/moment of momentum with respect to a joint axis. Equivalent particles are conveniently chosen to locate at the positions of the connection joints and in terms of their Cartesian coordinates the cut-joint constraint equations are easily formulated. The cut-joints kinematical constraints substitute for the unknown cut-joints constraint reaction forces that appear explicitly in the linear and angular momentum equations generated recursively along the separated serial chains.

It should be noted that in this formulation, the kinematical constraints due to some common types of kinematical joints (e.g. revolute or spherical joints) can be automatically eliminated by properly locating the equivalent particles. The remaining kinematical constraints along with the geometric constraints are, in general, either linear or quadratic in the Cartesian coordinates of the particles. Therefore, the coefficients of their Jacobian matrix are constants or linear in the rectangular Cartesian coordinates. Whereas in the formulation based on the relative coordinates (Denavit and Hartenberg, 1955), the constraint equations are derived based on loop closure equations which have the disadvantage that they do not directly determine the positions of the links and points of interest which makes the establishment of the dynamic problem more difficult. Also, the resulting constraint equations are highly nonlinear and contain complex circular functions. The absence of these circular functions in the point coordinate formulation leads to faster convergence and better accuracy. Furthermore, preprocessing the mechanism by the topological graph theory is not necessary as it would be the case with loop constraints.

Also, in comparison with the absolute coordinates formulation, the manual work of the local axes attachment and local coordinates evaluation as well as the use of the rotational variables and the rotation matrices in the absolute coordinate formulation are not required in the point coordinate formulation. This leads to fully computerized analysis and accounts for a reduction in the computational time and memory storage. In addition to that, the constraint equations take much simpler forms as compared with the absolute coordinates.
The elimination of the rotational coordinates, angular velocities and angular accelerations in the presented formulation, leads to possible savings in computation time when this procedure is compared against the absolute or relative coordinate formulation. It has been determined that numerical computations associated with rotational transformation matrices and their corresponding coordinate transformations between reference frames is time consuming and, therefore, if these computations are avoided more efficient codes may be developed (Nikravesh and Attia, 1994). The elimination of rotational coordinates can also be found very beneficial in design sensitivity analysis of multibody systems. In most procedures for design sensitivity analysis, leading to an optimal design process, the derivatives of certain functions with respect to a set of design parameters are required. Analytical evaluation of these derivatives are much simpler if the rotational coordinates are not present and if we only deal with translational coordinates.

Some practical applications of multibody dynamics require one or more bodies in the system to be described as deformable in order to obtain a more realistic dynamic response (Nikravesh and Attia, 1994). Deformable bodies are normally modeled by the finite element technique. Assume that the deformable body is connected to a rigid body described by a set of particles. Then, one or more particles of the rigid body can coincide with one or more nodes of the deformable body in order to describe the kinematical joint between the two bodies. This is a much simpler process that when the rigid body is described by a set of translational and rotational coordinates. In general, the point coordinates have additional advantages over the other systems of coordinates since they are the most suitable coordinates for the graphics routines and the animation programs.

Also, since we are dealing in this formulation with a system of particles instead of rigid bodies, therefore only the laws of particle dynamics are utilized in generating the equations of motion of the mechanical system. This makes the formulation much simpler than the other dynamic formulations which use the rigid body dynamical equations of motion both translational and rotational. In summary, the methodologies presented in this paper have many interesting characteristics which may be found useful in some applications. These methodologies can be combined with other methods to develop even more efficient, accurate, and flexible procedures. It should be noted that there is no single multibody formulation to be considered as the best formulation for general multibody dynamics. Each formulation has its own unique or common features and, therefore, selected features should be adopted to our advantages (Nikravesh and Attia, 1994).

\section{Dynamic Simulation of a Single Pendulum With a Rotating} End

Shown in Fig. 4 is a system consisting of two rigid bodies which are connected by a revolute joint and hung with a spherical joint to form a single pendulum with a rotating end. The system is an example of an open loop system with one branch and four degrees of freedom. Table 1 presents the inertia characteristics of the rigid bodies. Each rigid body is replaced with the four-primary particles representation. Particle 1 and 2 are chosen to lie along the axis of the revolute joint connecting the two bodies and therefore, they become common particles for the two bodies. Further, particles ( 3 and 4 ) and particles ( 5 and 6 ) are added, respectively, to body 1 and 2 such that the set of the 4 primary particles belongs to body $1(1,2$, 3 , and 4 ) and that belongs to body $2(1,2,5$, and 6 ) each does not lie in the same plane. Therefore, for a single rigid body, the positions of the primary particles can be freely chosen (not all lie in the same plane) but their masses together with the masses of the secondary 
particles must be determined in such a way that the rigid body and its equivalent system of particles have the same inertia characteristics. As a result of sharing the common particles 1 and 2, a total system of six particles replaces the whole system as shown in Fig. 4. The equations of motion are generated recursively along every branch as discussed in Sec. 4 and used to simulate the free response of the system.

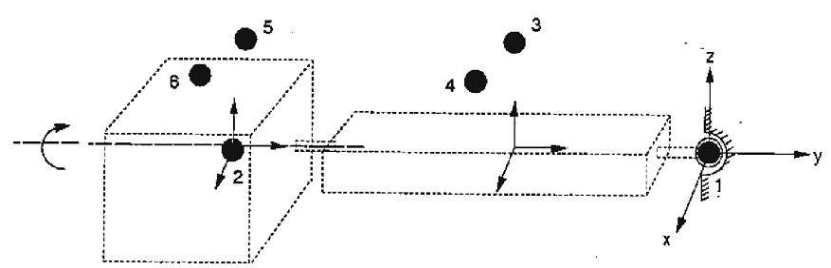

Figure 4. Schematic diagram of the double pendulum with the primary particles

Table 1. Description of the rigid bodies.

\begin{tabular}{|l|l|l|}
\hline Body\# & Mass $(\mathrm{kg})$ & $\begin{array}{l}\text { Inertia }\left(\mathrm{kg} . \mathrm{m}^{2}\right) \\
\xi \xi, \eta \eta, \zeta \zeta, \eta \zeta, \xi \zeta, \xi \eta\end{array}$ \\
\hline 1 & 1.5 & $0.1,0.1,0.1,0.0,0.0,0.0$ \\
\hline 2 & 25.0 & $25.0,25.0,25.0,0.0,0.0,0.0$ \\
\hline
\end{tabular}

The system starts motion from rest in the horizontal position while an initial angular velocity $2 \mathrm{rad} / \mathrm{S}$ is given to body 2 . The system has a regular precession motion due to the initially given angular velocity. Figure 5a-c presents the time variations of the $x$-, $y$-, and $z$ - coordinates of particle 2, respectively. The comparison with DAP-3D program, which is based on the absolute coordinates (Nikravesh, 1988), shows an excellent agreement between the results of the simulation.

\section{Conclusions}

In the present work, the dynamic simulation of constrained mechanical systems that are interconnected of rigid bodies is studied using a recursive projection algorithm. The concepts of linear and angular momentums are used to formulate the rigid body dynamical equations of motion which are expressed in terms of the rectangular Cartesian coordinates of a dynamically equivalent constrained system of particles. This groups the advantages of the automatic elimination of the unknown internal constraint forces, the absence of any rotational coordinates in addition to the rotational transformation matrices, and the elimination of the necessity to distribute the external forces and force couples over the particles. Also, the formulation can be considered as a natural extension to the finite element representation for a deformable body. Some useful geometric relations are used which result in a reduced system of differential-algebraic equations. The formulation can be applied to open and/or closed-chain with the common types of kinematical joints. The developed formulation with its interesting characteristics may be found useful in some applications.

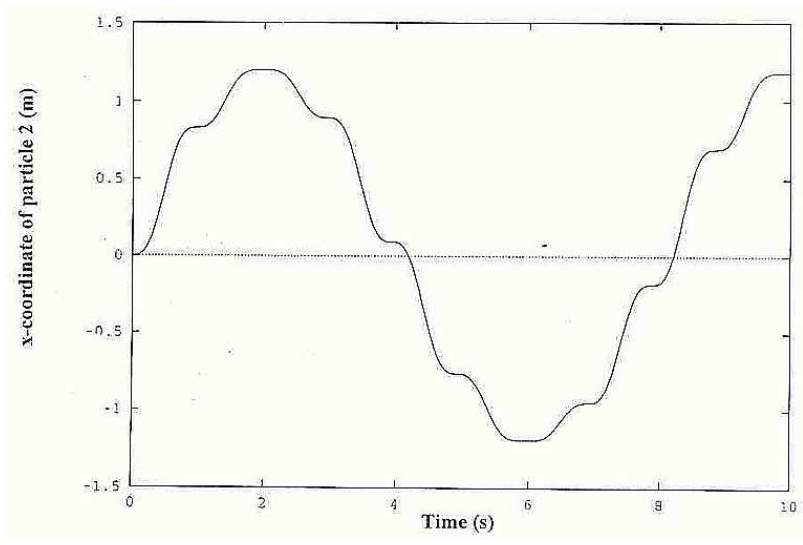

Figure 5a. The time variation of the $\mathrm{x}$-coordinate of particle 2.

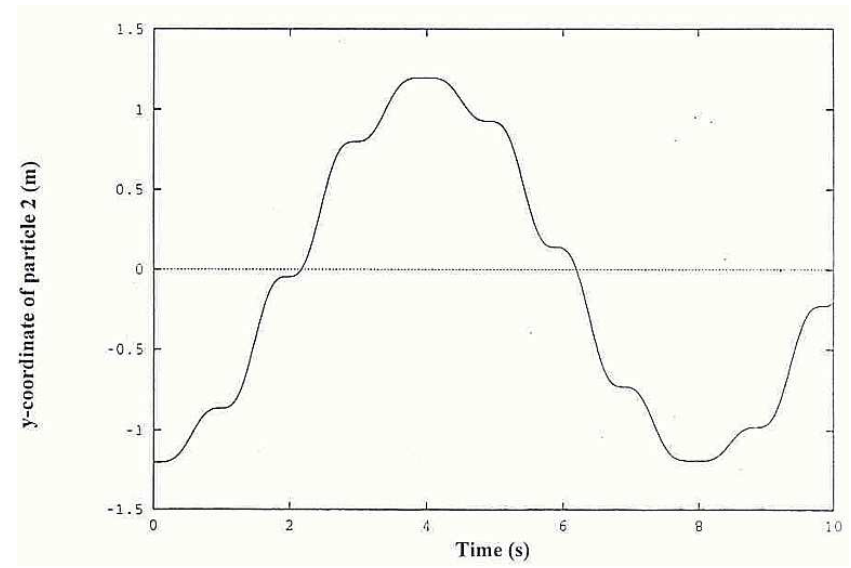

Figure $5 \mathrm{~b}$. The time variation of the $y$-coordinate of particle 2.

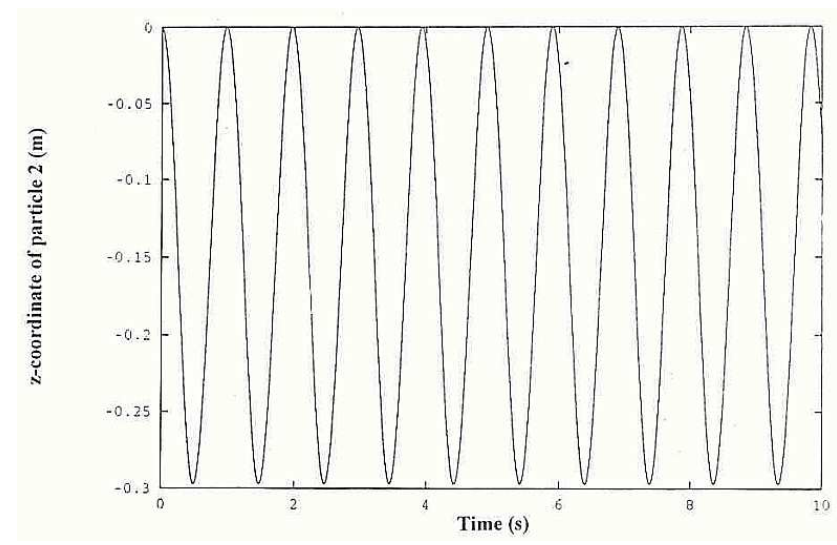

Figure $5 \mathrm{c}$. The time variation of the z-coordinate of particle 2.

\section{References}

Attia, H.A., 1993, A Computer-Oriented Dynamical Formulation with Applications to Multibody Systems, Ph.D. Dissertation, Department of Engineering Mathematics and Physics, Faculty of Engineering, Cairo University.

Attia, H.A., 1998, "Formulation of the equations of motion for the RRRR robot manipulator", Transactions of the Canadian Society for Mechanical Engineers, vol. 22, No. 1, pp. 83-93. 
Attia, H.A., 2004, "Equations of motion of planar mechanical systems based on particle dynamics and a recursive algorithm", Proceedings of the Institution of Mechanical Engineers (IMechE) Part K: Journal of Multi-body Dynamics, Vol. 218, No. 1, pp. 31-38.

Denavit, J., Hartenberg, R.S., 1955, "A Kinematic Notation for LowerPair Mechanisms Based on Matrices", ASME Journal of Applied Mechanics, pp. 215-221.

De Jalon, J.G., Unda, J., Avello, A., and Jimenez, J.M., 1986, "Dynamic analysis of three-dimensional mechanisms in 'Natural' coordinates", ASME Paper No. 86-DET-137.

De Jalon, J.G. and Bayo, E., 1994, "Kinematic and dynamic simulation of multibody systems", Springer-Verlag.

Goldstein, H., 1950, Classical mechanics, Addison-Wesley, Reading, Mass.

Gear, C.W., 1988, "Differential-algebraic equations index transformations", SIAM Journal of Scientific and Statistical Computing 9, pp. 39-47.

Kim S.S., And Vanderploeg, M.J., 1968, "A General and Efficient Method for Dynamic Analysis of Mechanical Systems Using Velocity
Transformation", ASME Journal of Mechanisms, Transmissions and Automation in Design, Vol. 108, No. 2, pp. 176-182, 1986.

Nikravesh P.E., 1988, Computer Aided Analysis of Mechanical Systems, Prentice-Hall, Englewood Cliffs, N.J..

Nikravesh, P.E., and Gim, G., 1989, "Systematic Construction of the Equations of Motion for Multibody Systems Containing Closed Kinematic Loop", ASME Design Conference.

Nikravesh, P.E. and Attia, H.A., 1994, "Construction of the equations of motion for multibody dynamics using point and joint coordinates," Computer-Aided Analysis of Rigid and Flexible Mechanical Systems, Kluwer Academic Publications, NATO ASI, Series E: Applied Sciences-vol. 268, pp. 31-60.

Orlandea, N., Chace, M.A., And Calahan, D.A., 1977, "A SparsityOriented Approach to Dynamic Analysis and Design of Mechanical Systems, Part I and II", ASME Journal of Engineering for Industry, Vol. 99, pp. 773-784.

Sheth P.N. and Uicker, Jr.J.J., 1972, "IMP (Integrated Mechanisms Program), A Computer-Aided Design Analysis System for Mechanisms Linkages", ASME Journal of Engineering for Industry, Vol. 94, pp. 454-468. 